\title{
Chaos Control of a Fractional-Order Financial System
}

\author{
Mohammed Salah Abd-Elouahab, ${ }^{1}$ Nasr-Eddine Hamri, ${ }^{1}$ \\ and Junwei Wang ${ }^{2}$ \\ ${ }^{1}$ Department of Science and Technology, University Center of Mila, Mila 43000, Algeria \\ ${ }^{2}$ Cisco School of Informatics, Guangdong University of Foreign Studies, Guangzhou 510006, China
}

Correspondence should be addressed to Mohammed Salah Abd-elouahab, medsalah3@yahoo.fr

Received 4 November 2009; Revised 29 April 2010; Accepted 2 June 2010

Academic Editor: Carlo Cattani

Copyright (C) 2010 Mohammed Salah Abd-elouahab et al. This is an open access article distributed under the Creative Commons Attribution License, which permits unrestricted use, distribution, and reproduction in any medium, provided the original work is properly cited.

Fractional-order financial system introduced by W.-C. Chen (2008) displays chaotic motions at order less than 3. In this paper we have extended the nonlinear feedback control in ODE systems to fractional-order systems, in order to eliminate the chaotic behavior. The results are proved analytically by applying the Lyapunov linearization method and stability condition for fractional system. Moreover numerical simulations are shown to verify the effectiveness of the proposed control scheme.

\section{Introduction}

Nonlinear chaotic systems have attracted more attention of researchers in various fields of natural sciences. This is because these systems are rich in dynamics, and possess great sensitivity to initial conditions. Since the chaotic phenomenon in economics was first found in 1985 , great impact has been imposed on the prominent western economics at present, because the chaotic phenomenon's occurring in the economic system means that the macroeconomic operation has in itself the inherent indefiniteness. Although the government can adopt such macrocontrol measures as the financial policies or the monetary policies to interfere, the effectiveness of the interference is very limited. The instability and complexity make the precise economic prediction greatly limited, and the reasonable prediction behavior has become complicated as well. In the fields of finance, stocks, and social economics, because of the interaction between nonlinear factors, with all kinds of economic problems being more and more complicated and with the evolution process from low dimensions to high dimensions, the diversity and complexity have manifested themselves in the internal 
structure of the system and there exists extremely complicated phenomenon and external characteristics in such a kind of system. So it has become more and more important to study the control of the complicated continuous economic system, and stabilize the instable periodic or stationary solutions, in order to make the precise economic prediction possible $[1,2]$.

Great interest has been paid to the application of fractional calculus in physics, engineering systems, and even financial analysis [3,4]. The fact that financial variables possess long memories makes fractional modelling appropriate for dynamic behaviors in financial systems. Moreover, the control and synchronization of fractional-order dynamic systems is also performed by various researchers [5-10]. Fractional-order financial system proposed by Chen in [11] displays many interesting dynamic behaviors, such as fixed points, periodic motions, and chaotic motions. It has been found that chaos exists in this system with orders less than 3, period doubling, and intermittency routes to chaos were found. In this paper, we propose to eliminate the chaotic behaviors from this system, by extending the nonlinear feedback control in ODE systems to fractional-order systems. This paper is organized as follows. In Section 2, we present the financial system and its fractional version. In Section 3 general approach to feedback control scheme is given, and then we have extended this control scheme to fractional-order financial system, numerical results are shown. Finally, in Section 4 concluding comments are given.

\section{Financial System}

\subsection{Integer-Order Financial System}

Recently, the studies in $[1,2]$ have reported a dynamic model of finance, composed of three first-order differential equations. The model describes the time-variation of three state variables: the interest rate $x$, the investment demand $y$, and the price index $z$. The factors that influence the changes of $x$ mainly come from two aspects: firstly, it is the contradiction from the investment market, (the surplus between investment and savings); secondly, it is the structure adjustment from goods prices. The changing rate of $y$ is in proportion with the rate of investment, and in proportion by inversion with the cost of investment and the interest rate. The changes of $z$, on one hand, are controlled by the contradiction between supply and demand of the commercial market, and on the other hand, are influenced by the inflation rate. Here we suppose that the amount of supplies and demands of commercials is constant in a certain period of time, and that the amount of supplies and demands of commercials is in proportion by inversion with the prices. However, the changes of the inflation rate can in fact be represented by the changes of the real interest rate and the inflation rate equals the nominal interest rate subtracts the real interest rate. The original model has nine independent parameters to be adjusted, so it needs to be further simplified. Therefore, by choosing the appropriate coordinate system and setting an appropriate dimension to every state variable, we can get the following more simplified model with only three most important parameters:

$$
\begin{gathered}
\dot{x}=z+(y-a) x, \\
\dot{y}=1-b y-x^{2}, \\
\dot{z}=-x-c z,
\end{gathered}
$$


where $a \geq 0$ is the saving amount, $b \geq 0$ is the cost per investment, and $c \geq 0$ is the elasticity of demand of commercial markets. It is obvious that all three constants, $a, b$, and $c$, are nonnegative, For more detail about the study of the local topological structure and bifurcation of this system; see [1,2]. We assume that $a$ is control parameter and $b=0.1, c=1$.

\subsubsection{Analysing the System}

(i) If $a \geq 9$, system (2.1) has one fixed point:

$$
p_{1}=(0,10,0) \text {. }
$$

(ii) If $a<9$, system (2.1) has three fixed points:

$$
p_{1}=(0,10,0), \quad p_{2,3}=\left(\mp \sqrt{\frac{9-a}{10}}, a+1, \pm \sqrt{\frac{9-a}{10}}\right) .
$$

To study the stability of equilibrium points we apply the Lyapunov's first (indirect) method [12] so we have the following theorem.

Theorem 2.1. Let $x=x^{*}$ be an equilibrium point of a nonlinear system:

$$
\dot{x}=f(x),
$$

where $f: D \rightarrow \mathbb{R}^{n}$ is continuously differentiable and $D \subset \mathbb{R}^{n}$ is the neighborhood of the equilibrium point $x^{*}$. Let $\lambda_{i}$ denote the eigenvalues of the Jacobian matrix $A=\partial f /\left.\partial x\right|_{x^{*}}$ then the following are considered.

(i) If $\operatorname{Re} \lambda_{i}<0$ for all $i$, then $x=x^{*}$ is asymptotically stable.

(ii) If $\operatorname{Re} \lambda_{i}>0$ for one or more $i$, then $x=x^{*}$ is unstable.

(iii) If $\operatorname{Re} \lambda_{i} \leq 0$ for all $i$ and at least one $\operatorname{Re} \lambda_{j}=0$, then $x=x^{*}$ may be either stable, asymptotically stable, or unstable.

Since $A$ is only defined at $x^{*}$, stability determined by the indirect method is restricted to infinitesimal neighborhoods of $x^{*}$.

To study the signs of the real parts of eigenvalues, we have the following famous criterion [13]. 
Criterion 1 (Routh-Hurwitz). Given the polynomial $P(\lambda)=\lambda^{n}+a_{1} \lambda^{n-1}+\cdots+a_{n-1} \lambda+a_{n}$, where the coefficients $a_{i}, i=1,2, \ldots, n$, are real constants, define the $n$ Hurwitz matrices

$$
\begin{aligned}
H_{1} & =\left(a_{1}\right), \\
H_{2} & =\left(\begin{array}{cc}
a_{1} & 1 \\
a_{3} & a_{2}
\end{array}\right) \\
\vdots & \\
H_{n} & =\left(\begin{array}{cccccc}
a_{1} & 1 & 0 & 0 & \cdots & 0 \\
a_{3} & a_{2} & a_{1} & 1 & \cdots & 0 \\
a_{5} & a_{4} & a_{3} & a_{2} & \cdots & 0 \\
\vdots & \vdots & \vdots & \vdots & \cdots & \vdots \\
0 & 0 & 0 & 0 & \cdots & a_{n}
\end{array}\right)
\end{aligned}
$$

where $a_{i}=0$ if $i>n$.

All of roots of the polynomial have negative real part if and only if the determinants of all Hurwitz matrices are positive: $\operatorname{det} H_{i}>0, i=1,2, \ldots, n$.

Routh-Hurwitz criteria for $n=3$ are $a_{1}>0, a_{3}>0$ and $a_{1} a_{2}-a_{3}>0$.

\section{Stability of $p_{1}$}

The Jacobian matrix of system (2.1) at the equilibrium point $p_{1}$ is

$$
J_{p_{1}}=\left(\begin{array}{ccc}
10-a & 0 & 1 \\
0 & -\frac{1}{10} & 0 \\
-1 & 0 & -1
\end{array}\right) \text {, }
$$

its characteristic polynomial is

$$
P(\lambda)=\lambda^{3}+\left(a-\frac{89}{10}\right) \lambda^{2}+\left(\frac{11 a-99}{10}\right) \lambda+\left(\frac{a-9}{10}\right) .
$$

By applying the Routh-Hurwitz criterion we find that the real parts of these eigenvalues are all negative if and only if

$$
\begin{gathered}
a-\frac{89}{10}>0, \\
a-9>0, \\
\left(a-\frac{89}{10}\right)\left(\frac{11 a-99}{10}\right)-\left(\frac{a-9}{10}\right)>0 .
\end{gathered}
$$




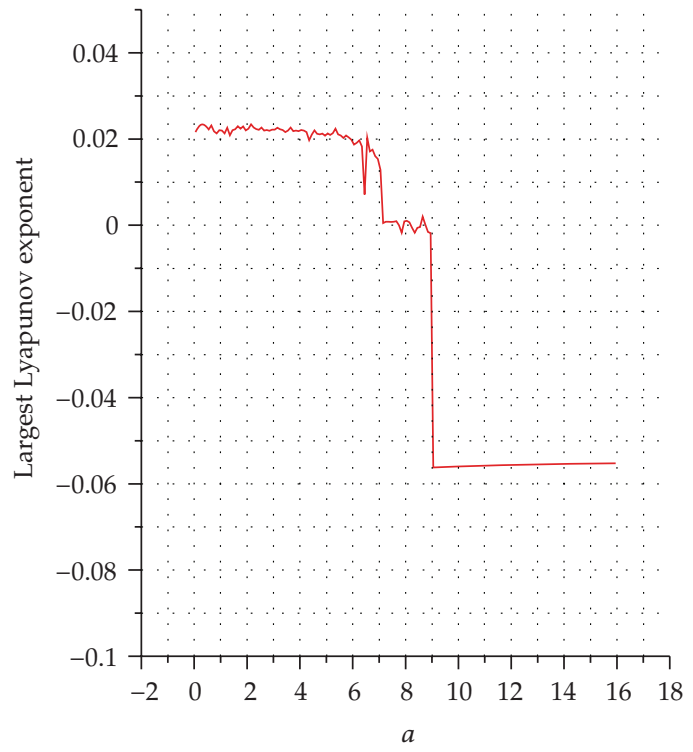

(a)

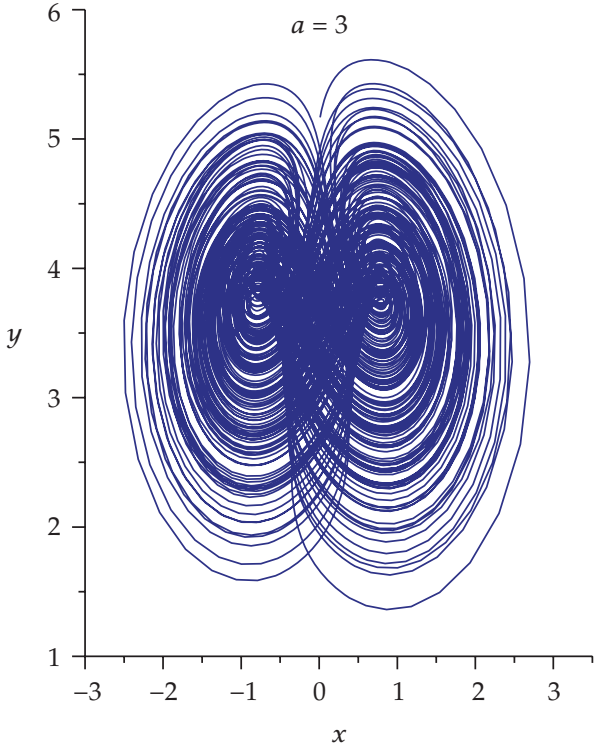

(b)

Figure 1: (a) Largest Lyapunov exponent according to $a$. (b) Chaotic attractor for $a=3$.

Then it follows that $a>9$, and thus $p_{1}$ is locally asymptotically stable if and only if $a>9$.

\section{Stability of $p_{2,3}$}

The Jacobian matrix of system (2.1) at the equilibrium points $p_{2,3}$ is

$$
J_{p_{2,3}}=\left(\begin{array}{ccc}
1 & \pm \sqrt{\frac{9-a}{10}} & 1 \\
\mp 2 \sqrt{\frac{9-a}{10}} & -0.1 & 0 \\
-1 & 0 & -1
\end{array}\right)
$$

and its characteristic polynomial is

$$
\tilde{p}(\lambda)=\lambda^{3}+\frac{1}{10} \lambda^{2}+\left(-\frac{1}{5} a+\frac{18}{10}\right) \lambda+\left(-\frac{1}{5} a+\frac{18}{10}\right) .
$$

The real parts of these eigenvalues are all negative if and only if

$$
\begin{gathered}
-\frac{1}{5} a+\frac{18}{10}>0 \\
\frac{1}{10}\left(-\frac{1}{5} a+\frac{18}{10}\right)-\left(-\frac{1}{5} a+\frac{18}{10}\right)>0 .
\end{gathered}
$$


Then it follows that

$$
\begin{aligned}
& a<9, \\
& a>9 .
\end{aligned}
$$

So $p_{2,3}$ are unstable for every value of $a$.

In order to detect the chaos we calculate the largest Lyapunov exponent $\lambda_{\max }$ using the scheme proposed by Wolf et al. [14]. The initial states are taken as $x(0)=2, y(0)=3, z(0)=2$, Figure 1(a) displays the evolution of $\lambda_{\max }$ according to $a$ and Figure 1(b) displays chaotic attractor for $a=3$. System (2.1) displays chaotic behavior in the windows $0<a<7\left(\lambda_{\max }>0\right)$, periodic behavior in $7 \leq a \leq 9\left(\lambda_{\max } \approx 0\right)$ and stationary behavior for $a>9\left(\lambda_{\max }<0\right)$.

\subsection{Fractional-Order Financial System}

Chen has introduced in [11] the generalization of system (2.1) for fractional incommensurateorder model which takes the form

$$
\begin{gathered}
D^{q_{1}} x=z+(y-a) x, \\
D^{q_{2}} y=1-b y-x^{2}, \\
D^{q_{3}} z=-x-c z .
\end{gathered}
$$

Fractional calculus is a generalization of ordinary differentiation and integration to arbitrary order but there are several definitions of fractional derivatives.

In this paper, we use the Caputo-type fractional derivative defined in [15] by:

$$
\begin{aligned}
D^{q} f(t) & =\frac{1}{\Gamma(n-q)} \int_{0}^{t}(t-\tau)^{n-q-1} f^{(n)}(\tau) d \tau \\
& =j^{n-q}\left(\frac{d^{n}}{d t^{n}} f(t)\right)
\end{aligned}
$$

where $n=[q]$ is the value of $q$ rounded up to the nearest integer, $\Gamma$ is the gamma function and $j^{\alpha}$ is the Riemann-Liouville integral operator defined by

$$
j^{\alpha} f(t)=\frac{1}{\Gamma(\alpha)} \int_{0}^{t}(t-\tau)^{\alpha-1} f(\tau) d \tau
$$

For the numerical solutions of system (2.13) we use the Adams-Bashforth-Moulton predictorcorrector scheme [16].

We assume that $q\left(q_{1}=q_{2}=q_{3}=q\right)$ is the control parameter, and $c=1, b=0.1, a=3$. Fractional system (2.13) has the same fixed points $p_{1,2,3}$ as integer system (2.1), but for the stability analysis we have this theorem introduced in $[17,18]$. 
Theorem 2.2. The fractional linear autonomous system

$$
\begin{aligned}
& D^{\alpha} X=A X \\
& X(0)=X_{0}
\end{aligned} \quad X \in \mathbf{R}^{n}, 0<\alpha<2, A \in \mathbf{R}^{n} \times \mathbf{R}^{n},
$$

is locally asymptotically stable if and only if

$$
\min _{i}\left|\arg \left(\lambda_{i}\right)\right|>\alpha \frac{\pi}{2}, \quad i=1,2, \ldots, n
$$

Proposition 2.3. Let $x=x^{*}$ be an equilibrium point of a fractional nonlinear system

$$
D^{\alpha} x=f(x), \quad 0<\alpha<2 .
$$

If the eigenvalues of the Jacobian matrix $A=\partial f /\left.\partial x\right|_{x^{*}}$ satisfy

$$
\min _{i}\left|\arg \left(\lambda_{i}\right)\right|>\alpha \frac{\pi}{2}, \quad i=1,2, \ldots, n,
$$

then the system is locally asymptotically stable at the equilibrium point $x^{*}$.

Proof. Let $x=x^{*}+\delta x$. Substituting in (2.18), we find

$$
D^{\alpha}\left(x^{*}+\delta x\right)=f\left(x^{*}+\delta x\right)
$$

so

$$
D^{\alpha}(\delta x)=f\left(x^{*}\right)+A \delta x+\bigcirc\left(\|\delta x\|^{2}\right) .
$$

Since $f\left(x^{*}\right)=0 \quad\left(x^{*}\right.$ is the equilibrium point of system (2.18)) and $\lim _{\|\delta x\| \rightarrow 0}\left(\bigcirc\left(\|\delta x\|^{2}\right) /\|\delta x\|\right)=0$, then

$$
D^{\alpha} \delta x \approx A \delta x
$$

Taking into account Theorem 2.2, we deduce that If the eigenvalues of the matrix $A$ satisfy

$$
\min _{i}\left|\arg \left(\lambda_{i}\right)\right|>\alpha \frac{\pi}{2}, \quad i=1,2, \ldots, n
$$

then $x^{*}$ is locally asymptotically stable.

This completes the proof. 
Stability of $p_{1}$

The Jacobian matrix of system (2.13) at the equilibrium point $p_{1}$ is

$$
J_{p_{1}}=\left(\begin{array}{ccc}
7 & 0 & 1 \\
0 & -\frac{1}{10} & 0 \\
-1 & 0 & -1
\end{array}\right) \text {, }
$$

and its characteristic polynomial is

$$
P(\lambda)=\lambda^{3}-\frac{59}{10} \lambda^{2}-\frac{66}{10} \lambda-\frac{6}{10} .
$$

its eigenvalues are $\lambda_{1} \approx-0.87298, \lambda_{2}=-1 / 10, \lambda_{3} \approx 6.8730$, we note that $\lambda_{3}$ is real positive then $\left|\arg \left(\lambda_{3}\right)\right|=0<q(\pi / 2)$, for all $\left.q \in\right] 0,2\left[\right.$, so $p_{1}$ is unstable for all $\left.q \in\right] 0,2[$.

\section{Stability of $p_{2,3}$}

The Jacobian matrix of system (2.13) at the equilibrium point $p_{2,3}$ is

$$
J_{p_{2,3}}=\left(\begin{array}{ccc}
1 & \pm \sqrt{\frac{3}{5}} & 1 \\
\mp 2 \sqrt{\frac{3}{5}} & -\frac{1}{10} & 0 \\
-1 & 0 & -1
\end{array}\right),
$$

its characteristic polynomial is

$$
\tilde{p}(\lambda)=\lambda^{3}+\frac{1}{10} \lambda^{2}+\frac{6}{5} \lambda+\frac{6}{5}
$$

and its eigenvalues are $\lambda_{1} \approx 0.31278+1.2474 i, \lambda_{2} \approx 0.31278-1.2474 i$, and $\lambda_{3} \approx-0.72556$, we have

$$
\left|\arg \left(\lambda_{1,2}\right)\right| \approx 1.3251, \quad\left|\arg \left(\lambda_{3}\right)\right|=\pi,
$$

so $\min _{i}\left|\arg \left(\lambda_{i}\right)\right| \approx 1.3251$, then the critical value of $q$ is

$$
q_{c}=\frac{2 \min _{i}\left|\arg \left(\lambda_{i}\right)\right|}{\pi} \approx 0.8436,
$$

(i) If $q<0.8436$, then $p_{2,3}$ are locally asymptotically stable. 


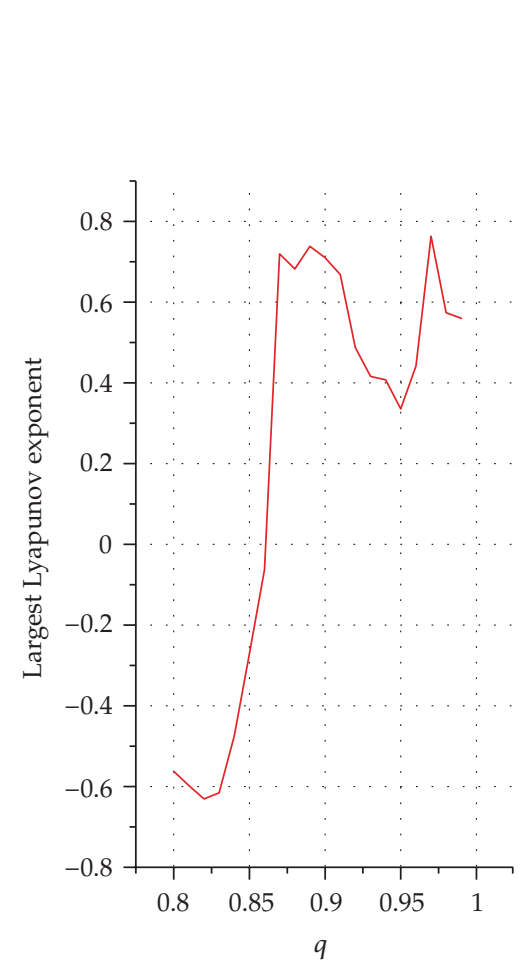

(a)
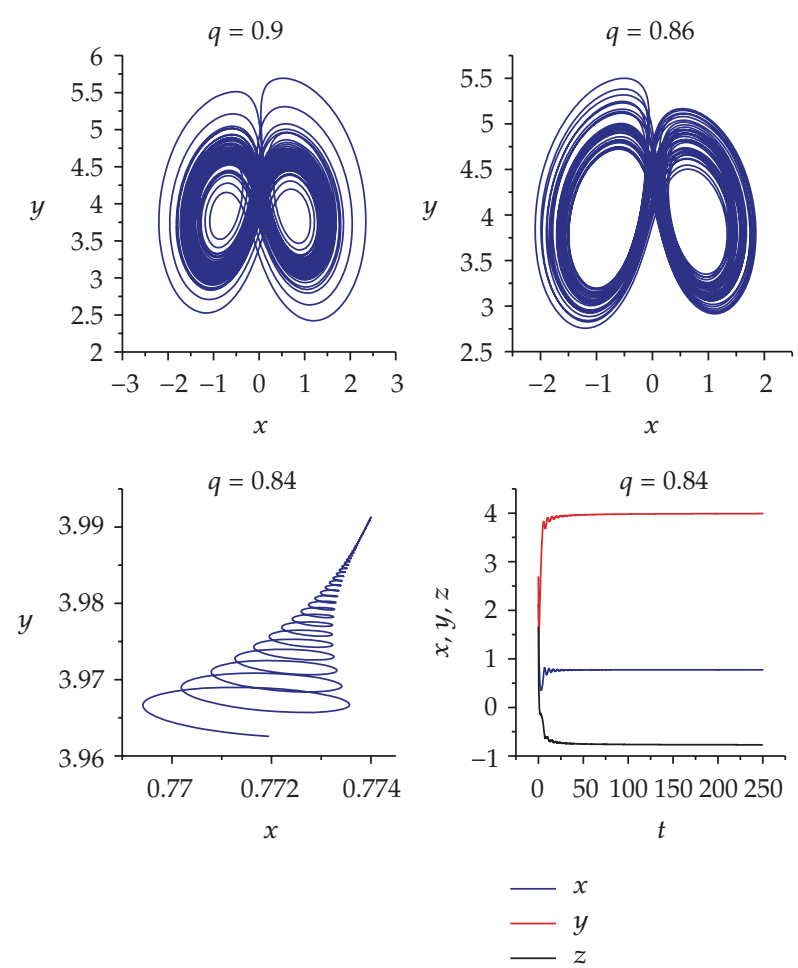

(b)

Figure 2: (a) Largest Lyapunov exponent according to $q$. (b) Phase diagram for some values of $q$.

(ii) If $q>0.8436$, then $p_{2,3}$ are unstable.

In order to detect the chaos, we calculate the largest Lyapunov exponent $\lambda_{\max }$.

The initial states are taken as $x(0)=2, y(0)=3, z(0)=2$, Figure 2(a) shows the evolution of $\lambda_{\max }$ according to $q$. System (2.13) exhibits chaotic behaviors for $q \geq 0.86$.

\section{Feedback Control}

\subsection{Integer Case}

A general approach to control a nonlinear dynamical system via feedback control can be formulated as follows:

$$
\dot{x}(t)=f(x, u, t),
$$

where $x(t)$ is the system state vector, and $u(t)$ the control input vector. Given a reference signal $\tilde{x}(t)$, the problem is to design a controller in the state feedback form:

$$
u(t)=g(x, t),
$$


where $g$ is vector-valued function, so that the controlled system

$$
\dot{x}(t)=f(x, g(x, t), t)
$$

can be driven by the feedback control $g(x, t)$ to achieve the goal of target tracking so we must have

$$
\lim _{t \rightarrow t_{f}}\|x(t)-\tilde{x}(t)\|=0
$$

Proposition 3.1. Let us consider the nonlinear system

$$
\dot{e}=F(e, t),
$$

where $e=x-\tilde{x}, \tilde{x}(t)$ is a periodic orbit (or fixed point) of the given system (3.1) with $u=0$, and $F(e, t)=f(x, g(x, t), t)-f(\tilde{x}, 0, t)$.

If 0 is a fixed point of system (3.5) and all eigenvalues of the jacobian matrix $A=\partial F /\left.\partial x\right|_{0}$ have negative real parts then the trajectory $x(t)$ of system (3.3) converge to $\tilde{x}(t)$

Proof. Since $\tilde{x}(t)$ is a periodic orbit (or fixed point) of the given system (3.1) with $u=0$, so it satisfies

$$
\dot{\tilde{x}}(t)=f(\tilde{x}, 0, t),
$$

a subtraction of (3.6) from (3.1) gives

$$
\dot{x}(t)-\dot{\tilde{x}}(t)=f(x, g(x, t), t)-f(\tilde{x}, 0, t),
$$

so

$$
\dot{e}=F(e, t) \text {. }
$$

Since all eigenvalues of the jacobian matrix $A$ have negative real parts, it follows from Theorem 2.1 that 0 is asymptotically stable, so we have $\lim _{t \rightarrow+\infty}\|e(t)\|=0$ then $\lim _{t \rightarrow+\infty} \| x(t)-$ $\tilde{x}(t) \|=0$, finally $x(t) \underset{t \rightarrow t_{f}}{\rightarrow} \tilde{x}(t)$.

\subsection{Fractional Case}

Let us consider the fractional system

$$
D^{\alpha} x(t)=f(x, u, t) .
$$

We proceed as in the integer case. the controlled system can be written as

$$
D^{\alpha} x(t)=f(x, g(x, t), t) .
$$




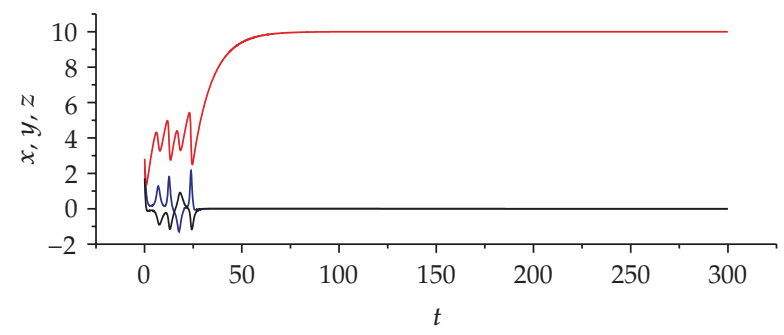

$$
\begin{array}{r}
x \\
y \\
-z
\end{array}
$$

(a)

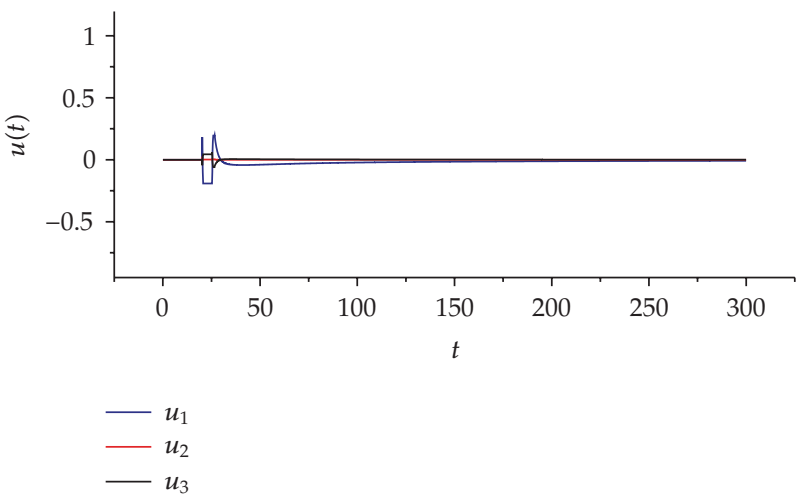

(b)

Figure 3: (a) Stabilizing the equilibrium point $p_{1}$ for $q=0.9$. (b) Evolution of the perturbation $u(t)$.

Let $\tilde{x}(t)$ be a periodic orbit (or fixed point) of the given system (3.9) with $u=0$, then we obtain the system error

$$
D^{\alpha} e(t)=F(e, t)
$$

Proposition 3.2. If 0 is a fixed point of system (3.11) and the eigenvalues of the jacobian matrix $A=\partial F /\left.\partial x\right|_{0}$ satisfies the condition

$$
\min _{i}\left|\arg \left(\lambda_{i}\right)\right|>\alpha \frac{\pi}{2}, \quad i=1,2, \ldots, n
$$

then the trajectory $x(t)$ of system (3.10) converge to $\tilde{x}(t)$.

Proof. It follows directly from Proposition 2.3. 


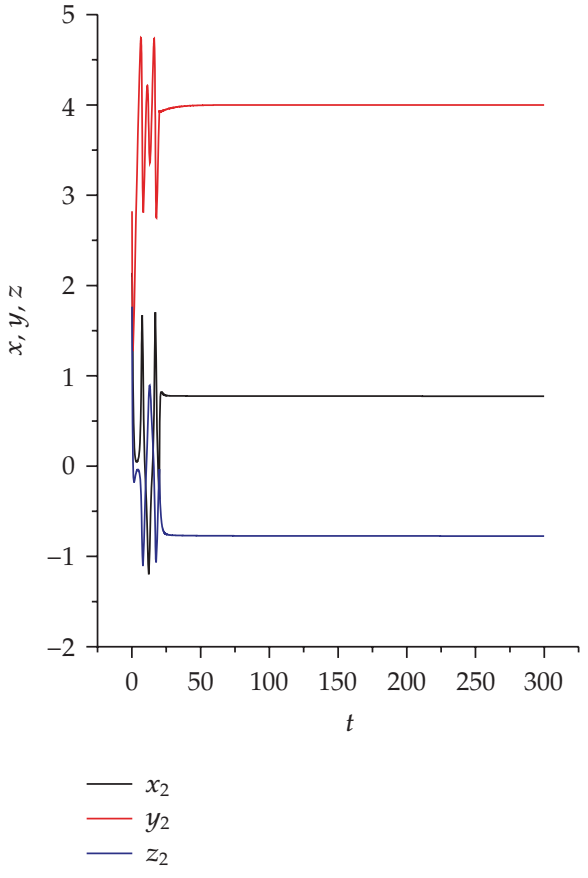

(a)

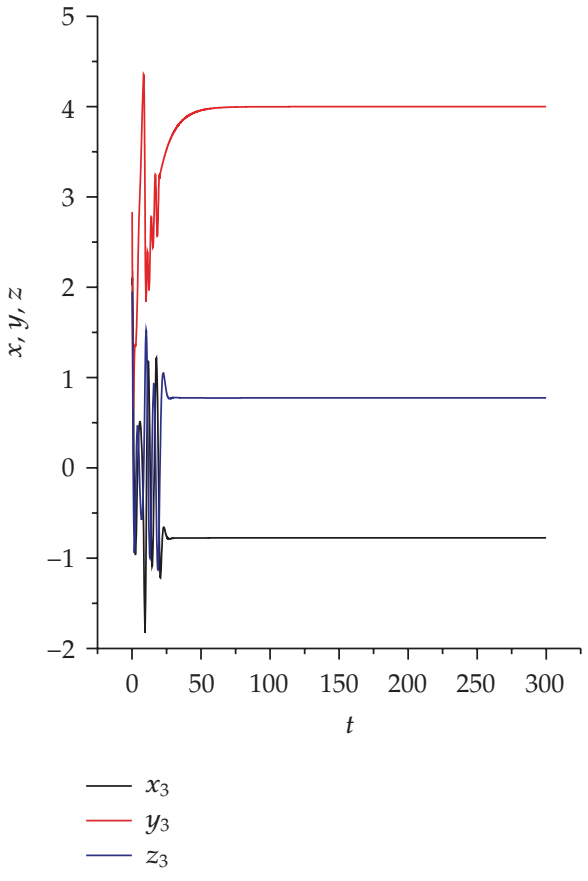

(b)

Figure 4: (a) Stabilizing the equilibrium point $p_{2}$ for $q=0.95$. (b) Stabilizing the equilibrium point $p_{3}$ for $q=1.4$.

\subsection{Application to the Fractional Financial System}

Let us consider the fractional financial system (2.13), we propose to stabilize unstable periodic orbit (or fixed point) $(\tilde{x}, \tilde{y}, \tilde{z})$, the controlled system is as follows:

$$
\begin{gathered}
D^{q_{1}} x=z+(y-a) x+u_{1}(t), \\
D^{q_{2}} y=1-b y-x^{2}+u_{2}(t), \\
D^{q_{3}} z=-x-c z+u_{3}(t) .
\end{gathered}
$$

Since $(\tilde{x}, \tilde{y}, \tilde{z})$ is solution of $(2.13)$, then we have:

$$
\begin{gathered}
D^{q_{1}} \tilde{x}=\tilde{z}+(\tilde{y}-a) \tilde{x}, \\
D^{q_{2}} \tilde{y}=1-b \tilde{y}-\tilde{x}^{2}, \\
D^{q_{3}} \tilde{z}=-\tilde{x}-c \tilde{z} .
\end{gathered}
$$




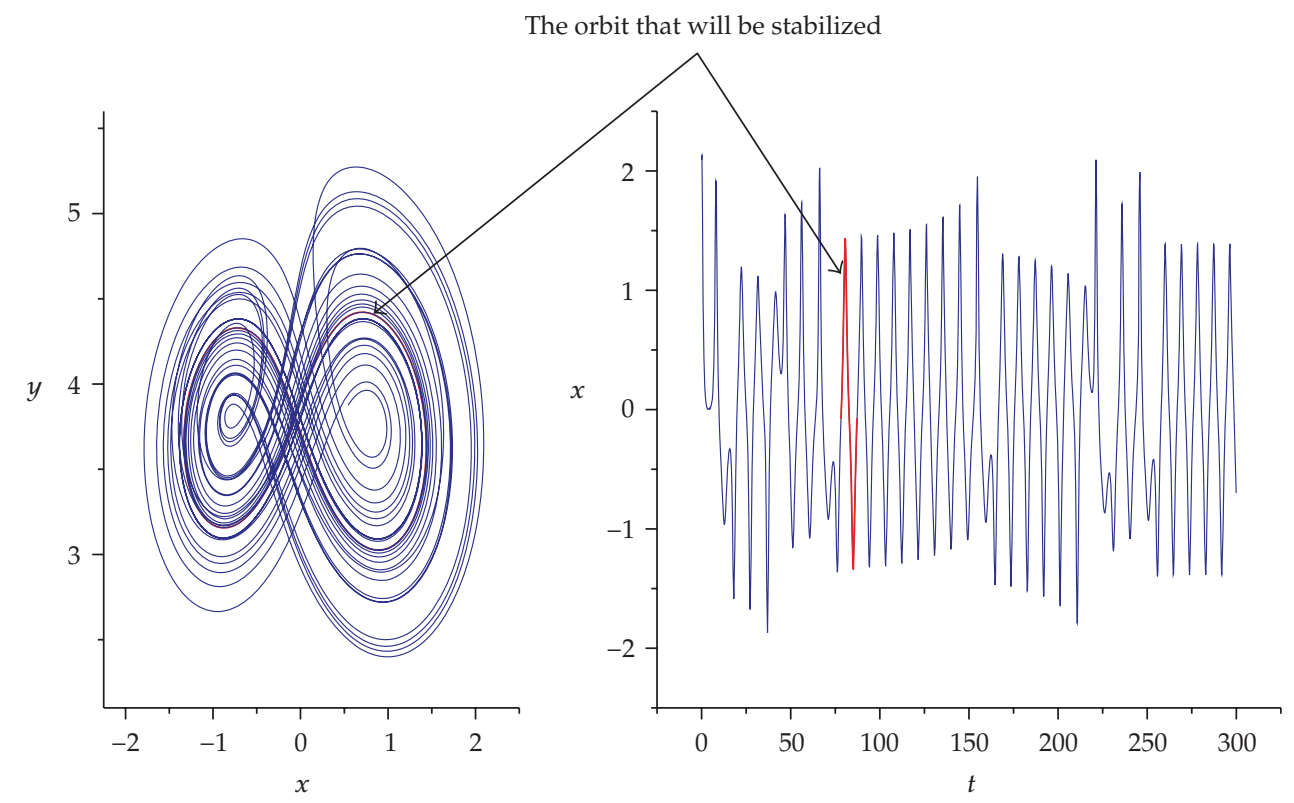

Figure 5: Selecting an unstable periodic orbit in the chaotic attractor of period $T=9$ for $q=0.97$.

Subtracting (3.14) from (3.13) with notation, $e_{1}=x-\tilde{x}, e_{2}=y-\tilde{y}, e_{3}=z-\tilde{z}$, we obtain the system error:

$$
\begin{gathered}
D^{q_{1}} e_{1}=e_{3}-a e_{1}+x y-\tilde{x} \tilde{y}+u_{1}(t), \\
D^{q_{2}} e_{2}=-b e_{2}-e_{1}(x+\tilde{x})+u_{2}(t), \\
D^{q_{3}} e_{3}=-e_{1}-c e_{3}+u_{3}(t) .
\end{gathered}
$$

We define the control functions as follow:

$$
\begin{gathered}
u_{1}(t)=-(x y-\tilde{x} \tilde{y}), \\
u_{2}(t)=e_{1}(x+\tilde{x}), \\
u_{3}(t)=e_{1} .
\end{gathered}
$$

So the system error (3.15) becomes

$$
\begin{gathered}
D^{q_{1}} e_{1}=e_{3}-a e_{1} \\
D^{q_{2}} e_{2}=-b e_{2}, \\
D^{q_{3}} e_{3}=-c e_{3} .
\end{gathered}
$$




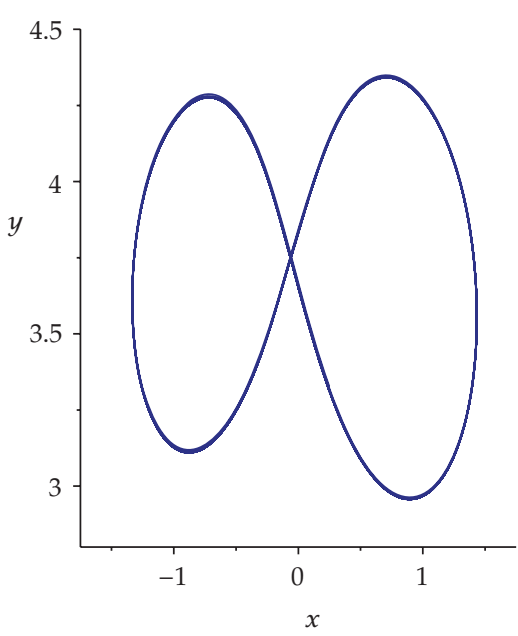

(a)

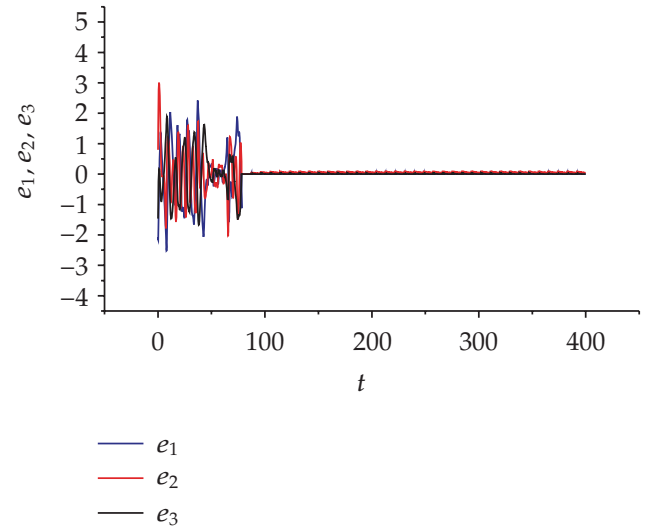

(b)
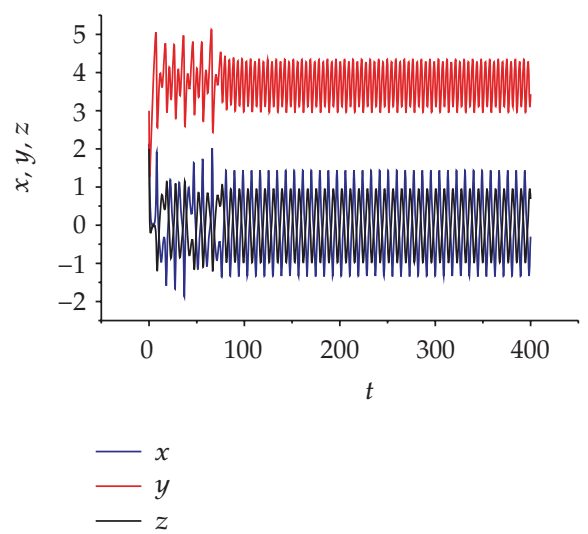

(c)

Figure 6: Stabilizing unstable periodic orbit of period $T=9$ for $q=0.97$.

The Jacobian matrix is $\left[\begin{array}{ccc}-a & 0 & 1 \\ 0 & -b & 0 \\ 0 & 0 & -c\end{array}\right]$ and its characteristic polynomial is:

$$
p(x)=x^{3}+(a+b+c) x^{2}+(a b+c(a+b)) x+a b c
$$

so we have the eigenvalues $\lambda_{1}=-a, \lambda_{2}=-b, \lambda_{3}=-c$. Since all eigenvalues are real negatives one has $\arg \left(\lambda_{i}\right)=\pi$, therefore $\left|\arg \left(\lambda_{i}\right)\right|>q(\pi / 2)$, for all $q$ satisfies $0<q<2$, it follows from Proposition 3.2 that the trajectory $x(t)$ of system (3.13) converges to $\tilde{x}(t)$ and the control is completed.

\subsection{Simulation Results}

In this section we give numerical results which prove the performance of the proposed scheme. As mentioned in Section 2.3 we have implemented the improved Adams-BashforthMoulton algorithm for numerical simulation. 


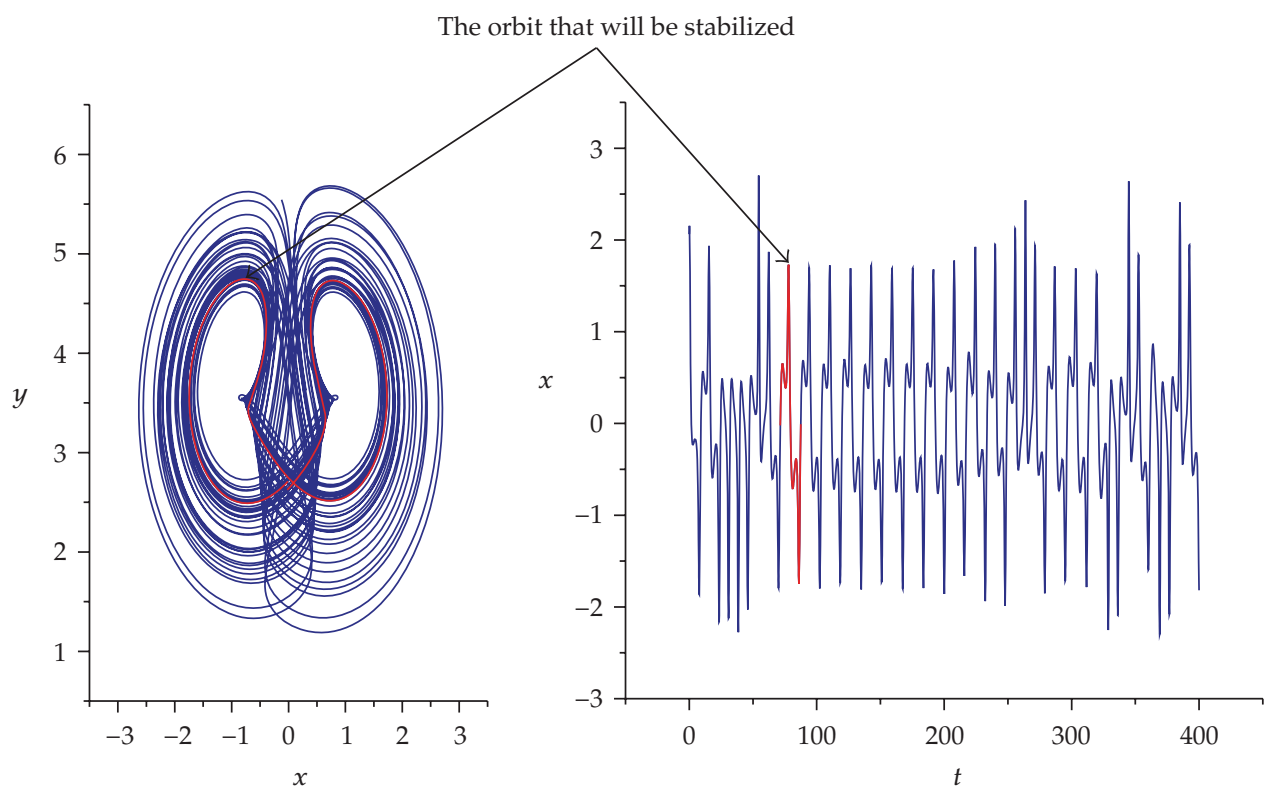

Figure 7: Selecting an unstable periodic orbit in the chaotic attractor of period $T=16.05$ for $q=1.1$.

The initial states are taken as $x(0)=2, y(0)=3, z(0)=2$.

\subsubsection{Stabilizing the Unstable Fixed Points}

The control can be started at any time according to our needs, so we choose to activate the control when $t \geq 20$, in order to make a comparison between the behavior before activation of control and after it.

For $q=0.9$ unstable point $p_{1}$ has been stabilized, as shown in Figure 3(a), note that $u_{1}(t)=-(x(t) y(t)-0 \times 10)=-x(t) y(t)$, so the control is activated when $t \geq 20$ and $|x(t) y(t)| \leq 0.2$ (more precisely $t=22.5$ ) in order to make the perturbation $u_{1}(t)$ smaller. firstly the evolution of $x(t), y(t), z(t)$ is chaotic, then when the control is started at $t=22.5$ we see that $p_{1}$ is rapidly stabilized.

In Figure 3(b) we observe the evolution of the perturbation $u(t)$, when the control is started we see that $u_{2}(t)$ and $u_{3}(t)$ are very small but $u_{1}(t)$ is a bit larger, after that the perturbation $u(t)$ becomes close to zero rapidly.

For $q=0.95$, the unstable point $p_{2}$ has been stabilized, as shown in Figure 4(a).

For $q=1.4$ the fixed point $p_{3}$ was stabilized, Figure $4(\mathrm{~b})$ shows the results of control.

When $t$ is less than 20, there is a chaotic behavior, but when the control is activated at $t=20$, the two points $p_{2}$ and $p_{3}$ are rapidly stabilized.

In the real world of finance if we want to have a good investment demand we can choose to stabilize $p_{1}$, and in this case the interest rate and price index will be near zero. During the recent financial crisis in 2009 many banks decided to reduce interest rates to nearly zero in order to control this situation. 


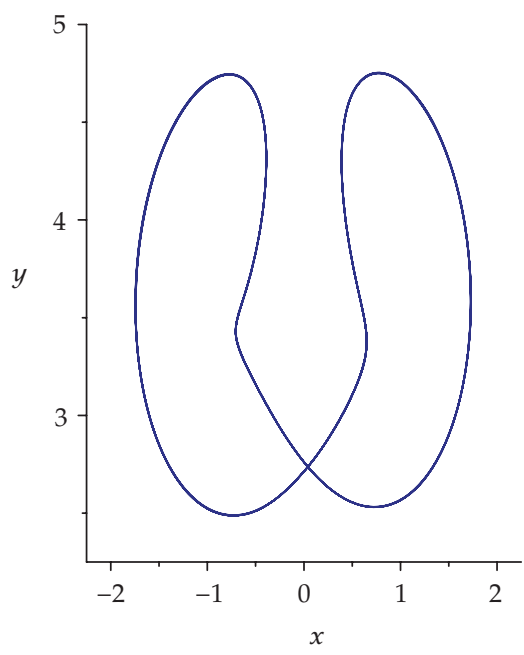

(a)
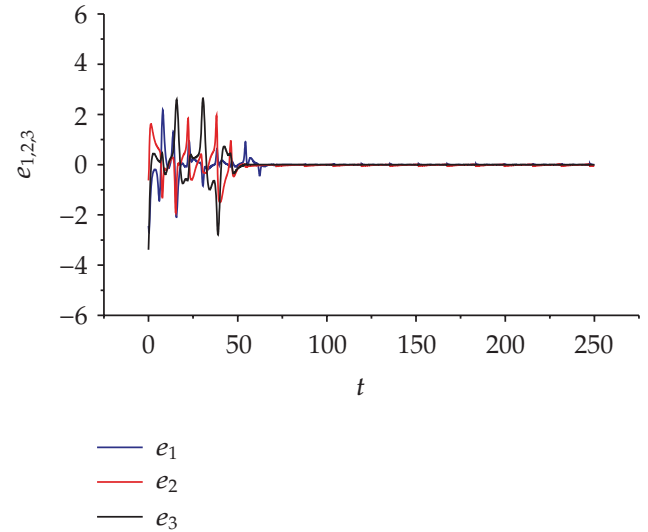

(b)

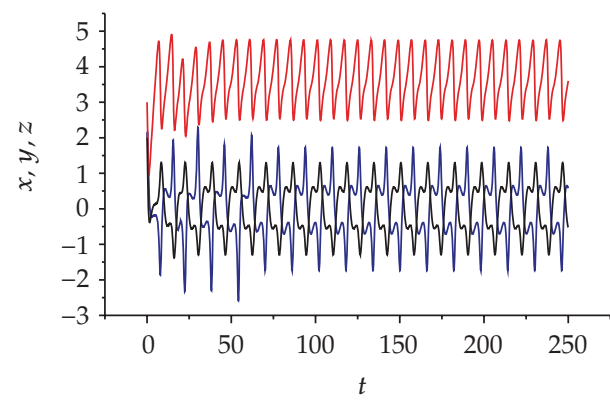

$x$
$-y$
$-z$

(c)

Figure 8: Stabilization an unstable periodic orbit of period $T=16.05$ for $q=1.1$.

\subsubsection{Stabilizing Unstable Periodic Orbit}

Although the unstable periodic orbits are dense in the chaotic attractor, we can choose one of them (which represent the performance of the system), by analyzing data experimental, after that we stabilize it. In this paper the close-return (CR) method [19] is used for the detection of UPO embedded in the attractor.

For $q=0.97$ we choose an unstable periodic orbit with period $T=9$, localized in the interval $[78.2,87.2]$ as shown in Figure 5, then the control is started at $t=87.2$, when the trajectory $x(t)$ begins to emerge from the unstable orbit, Figure 6 displays the results of control, if $t$ is less then 78.2 there is chaotic behavior (the error $e(t)$ is large), after the activation of control, this chaotic behavior is replaced by a periodic behavior and we note that the error $e(t)$ becomes very close to zero.

For $q=1.1$ we choose an unstable periodic orbit with period $T=16.05$, localized in the interval $[71.45,87.5]$ as shown in Figure 7, the control is started at $t=20$, Figure 8 displays the results of control. Although the control is executing at $t=20$, it does not give effect rapidly, 
and the orbit is stabilized at $t=63$, when the control is activated the error begins to diminish, and becomes close to zero after $t=63$.

The stabilization of the periodic orbits is very important, because it permits, on the one hand to make some predictions, and secondly, it is more realistic than the stabilization of the stationary points in the financial circle, where one cannot generally fix the interest rate and the investment demand as well as the price index, for a long period.

\section{Conclusions}

Chaotic phenomenon makes prediction impossible in the financial world; then the deletion of this phenomenon from fractional financial system is very useful, the main contribution of this paper is to this end.

Nonlinear feedback control scheme has been extended to control fractional financial system. The results are proved analytically by applying the Lyapunov linearization method and stability condition for fractional system. Numerically the unstable fixed points $p_{1,2,3}$ have been successively stabilized for different values of $q$; moreover unstable periodic orbit has stabilized. This proves the performance of the proposed scheme.

\section{References}

[1] J. H. Ma and Y. S. Chen, "Study for the bifurcation topological structure and the global complicated character of a kind of nonlinear finance system. I," Applied Mathematics and Mechanics, vol. 22, no. 11, pp. 1240-1251, 2001.

[2] J. H. Ma and Y. S. Chen, "Study for the bifurcation topological structure and the global complicated character of a kind of nonlinear finance system. II," Applied Mathematics and Mechanics, vol. 22, no. 12, pp. 1375-1382, 2001.

[3] I. Podlubny, Fractional Differential Equations: An Introduction to Fractional Derivatives, Fractional Differential Equations, to Methods of Their solution and Some of Their Application, vol. 198 of Mathematics in Science and Engineering, Academic Press, San Diego, Calif, USA, 1999.

[4] R. Hilfer, Ed., Applications of Fractional Calculus in Physics, World Scientific, River Edge, NJ, USA, 2000.

[5] A. Oustaloup, J. Sabatier, and P. Lanusse, "From fractal robustness to the CRONE control," Fractional Calculus \& Applied Analysis, vol. 2, no. 1, pp. 1-30, 1999.

[6] A. Oustaloup, F. Levron, B. Mathieu, and F. M. Nanot, "Frequency-band complex noninteger differentiator: characterization and synthesis," IEEE Transactions on Circuits and Systems I, vol. 47, no. 1, pp. 25-39, 2000.

[7] T. T. Hartley and C. F. Lorenzo, "Dynamics and control of initialized fractional-order systems," Nonlinear Dynamics, vol. 29, no. 1-4, pp. 201-233, 2002.

[8] J. Wang, X. Xiong, and Y. Zhang, "Extending synchronization scheme to chaotic fractional-order Chen systems," Physica A, vol. 370, no. 2, pp. 279-285, 2006.

[9] Z. M. Odibat, N. Corson, M. A. Aziz-Alaoui, and C. Bertelle, "Synchronization of chaotic fractionalorder systems via linear control," International Journal of Bifurcation and Chaos, vol. 20, no. 1, pp. 81-97, 2010.

[10] X.-y. Wang, Y.-j. He, and M.-j. Wang, "Chaos control of a fractional order modified coupled dynamos system," Nonlinear Analysis: Theory, Methods E Applications, vol. 71, no. 12, pp. 6126-6134, 2009.

[11] W.-C. Chen, "Nonlinear dynamics and chaos in a fractional-order financial system," Chaos, Solitons and Fractals, vol. 36, no. 5, pp. 1305-1314, 2008.

[12] A. M. Lyapunov, The General Problem of the Stability of Motion, Taylor \& Francis, London, UK, 1992.

[13] I. S. Gradshteyn and I. M. Ryzhik, Table of Integrals, Series, and Products, Translation edited and with a preface by A. Jeffrey and D. Zwillinger, Academic Press, San Diego, Calif, USA, 6th edition, 2000.

[14] A. Wolf, J. B. Swift, H. L. Swinney, and J. A. Vastano, “Determining Lyapunov exponents from a time series," Physica D, vol. 16, no. 3, pp. 285-317, 1985.

[15] M. Caputo, "Linear models of dissipation whose $Q$ is almost frequency independent-II," Geophysical Journal of the Royal Astronomical Society, vol. 13, no. 5, pp. 529-539, 1967. 
[16] K. Diethelm and A. D. Freed, "The FracPECE subroutine for the numerical solution of differential equations of fractional order," in Forschung und wissenschaftliches Rechnen, S. Heinzel and T. Plesser, Eds., pp. 57-71, Gesellschaft für Wisseschaftliche Datenverarbeitung, Gottingen, Germany, 1998.

[17] D. Matignon, "Stability properties for generalized fractional differential systems," in Systèmes différentiels fractionnaires, vol. 5 of ESAIM Proc., pp. 145-158, Soc. Math. Appl. Indust., Paris, France, 1998.

[18] M. Moze and J. Sabatier, "LMI tools for stability analysis of fractional systems," in Proceedings of ASME International Design Engineering Technical Conferences E Computers and Information in Engineering Conference, pp. 1-9, Long Beach, Calif, USA, September 2005.

[19] D. Auerbach, P. Cvitanović, J.-P. Eckmann, G. Gunaratne, and I. Procaccia, "Exploring chaotic motion through periodic orbits," Physical Review Letters, vol. 58, no. 23, pp. 2387-2389, 1987. 


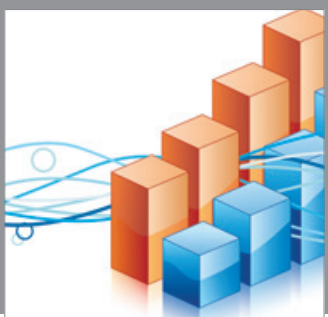

Advances in

Operations Research

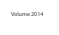

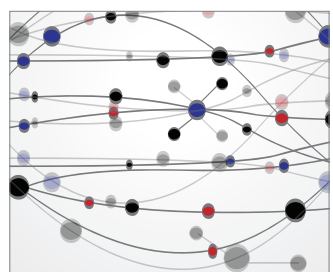

\section{The Scientific} World Journal
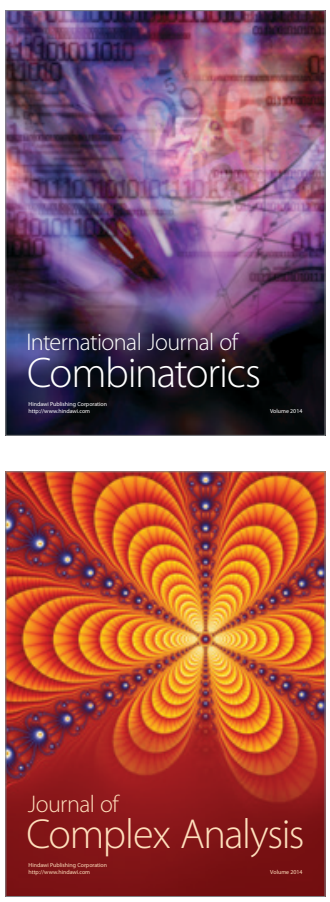

International Journal of

Mathematics and

Mathematical

Sciences
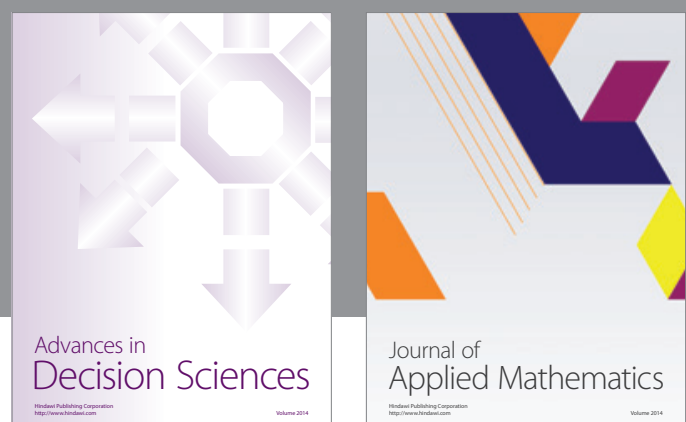

Journal of

Applied Mathematics
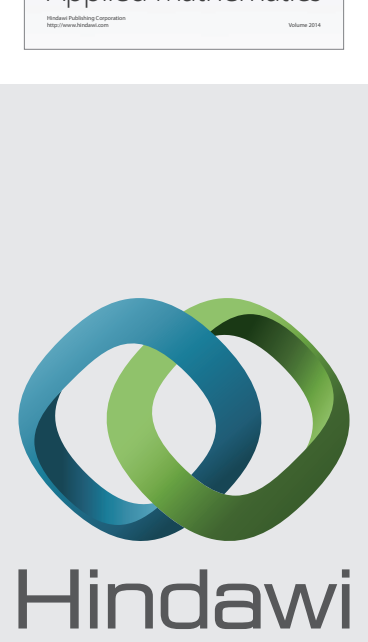

Submit your manuscripts at http://www.hindawi.com
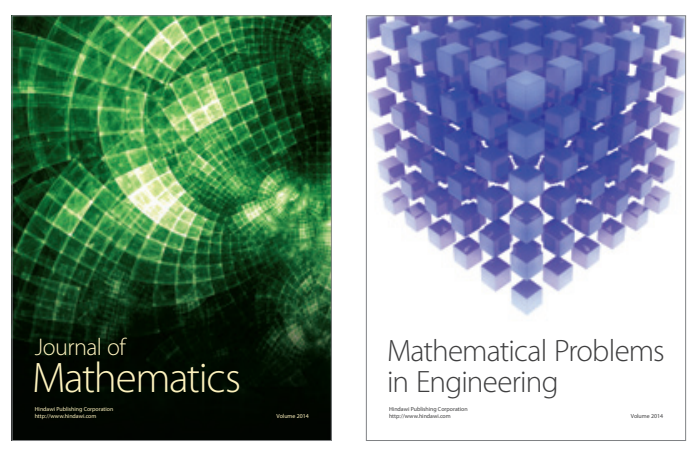

Mathematical Problems in Engineering
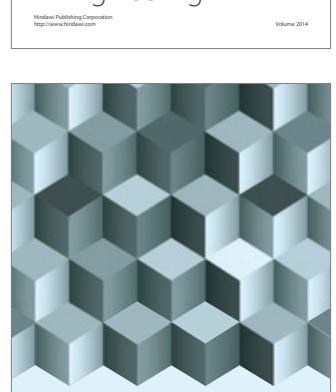

Journal of

Function Spaces
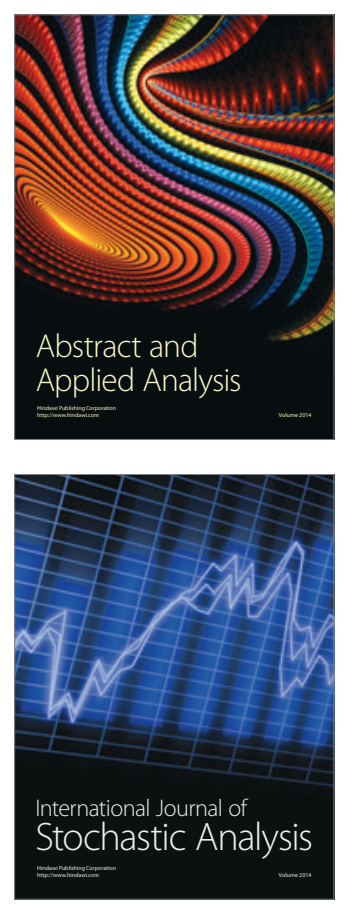

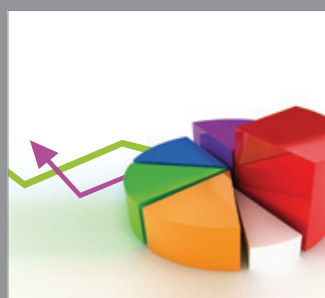

ournal of

Probability and Statistics

Promensencen
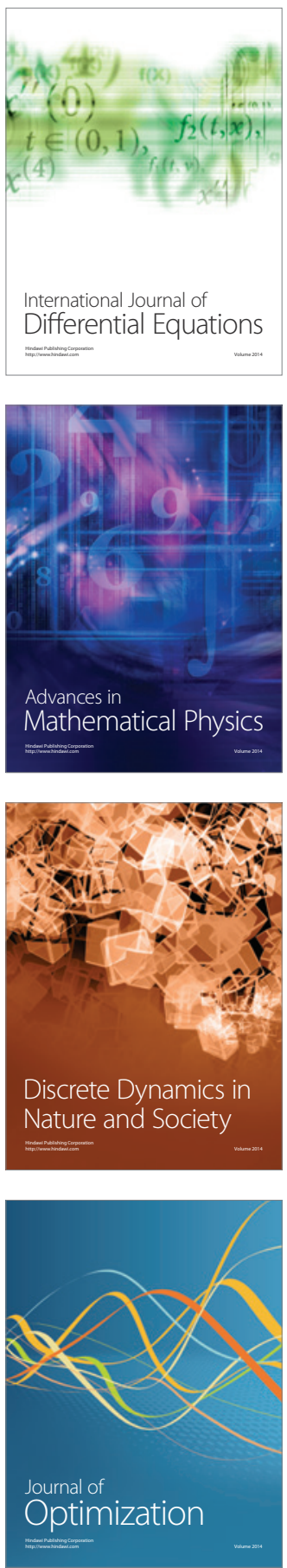